\title{
Chronic Alcoholic Liver Disease and Mortality Risk in Spontaneous Bacterial Peritonitis: Analysis of 6,530 Hospitalizations
}

\author{
Renu Bhandari ${ }^{1}$, Khalida Khaliq ${ }^{2}$, Virendrasinh Ravat ${ }^{3}$, Pawandeep Kaur ${ }^{4}$, Rikinkumar S. Patel ${ }^{5}$ \\ 1. Medicine, Manipal College of Medical Sciences, Kaski, NPL 2. Psychiatry/Medicine, North Tampa Behavioral Health, \\ Tampa, USA 3. Internal Medicine, Krishna Institute of Medical Sciences, Karad, IND 4. Medicine, Sri Guru Ram Das \\ Institute of Medical Sciences and Research, Amritsar, IND 5. Psychiatry, Griffin Memorial Hospital, Norman, USA
}

Corresponding author: Rikinkumar S. Patel, rpatel_09@arcadia.edu

\section{Abstract \\ Objective}

Our study aimed to assess the risk of in-hospital mortality due to chronic alcoholic liver disease (CALD) and other comorbidities in spontaneous bacterial peritonitis (SBP) inpatients.

\section{Methods}

We conducted a cross-sectional study using the Nationwide Inpatient Sample (NIS, 2012 to 2014) from the United States and included 6,530 patients (age 18-50 years) with a primary diagnosis of SBP. Logistic regression was used to evaluate the odds ratio (OR) for in-hospital mortality in SBP by comorbidities.

\section{Results}

The prevalence of CALD in SBP patients is $43.6 \%$, and a higher proportion were males (68.8\%) and whites (67\%). Middle-aged adults (OR 2.8, 95\% CI 1.74-4.45) had higher odds of in-hospital mortality in SBP patients. Race and sex were non-significant predictors for mortality risk. Patients with comorbid coagulopathy (OR 1.9, 95\% CI 1.45-2.48) and heart failure (OR 3.9, 95\% CI 2.46-6.36) have increased mortality in SBP inpatients. After controlling confounders, CALD was significantly associated with increased in-hospital mortality (OR 1.5, 95\% CI 1.12-1.94) in SBP inpatients.

\section{Conclusion}

CALD is an independent factor in increasing the risk of in-hospital mortality in SBP patients by $48 \%$. Alcohol use screening, and alcohol abstinence and supportive therapy need to be implemented at an earlier stage to improve health-related quality of life and reduce in-hospital mortality in SBP patients.

Received 05/04/2020

Review began 05/10/2020 Review ended 05/11/2020 Published 05/18/2020

() Copyright 2020 Bhandari et al. This is an open access article distributed under the terms of the Creative Commons Attribution License CC-BY 4.0., which permits unrestricted use, distribution, and reproduction in any medium, provided the original author and source are credited.
Categories: Internal Medicine, Psychiatry, Gastroenterology

Keywords: spontaneous bacterial peritonitis, alcohol-related liver disease, alcohol dependence, alcohol misuse, mortality risk

\section{Introduction}

Spontaneous bacterial peritonitis (SBP) is an infection of the ascitic fluid without any previous evidence of intra-abdominal surgery [1]. SBP is diagnosed based on polymorphonuclear neutrophils higher than 250 cells per cubic millimeter [2]. The prevalence of SBP in liver cirrhosis range from $11 \%$ to $14 \%$ and the incidence of SBP in hospitalized patients ranges from $10 \%$ to $30 \%[3,4]$. In earlier days, SBP was associated with a high mortality rate of about $70 \%$, but due to the advancement of treatment and diagnostic paracentesis, the mortality rate in hospitalized patients has decreased to around $17.6 \%$ [4]. Some of the studies have shown a higher mortality rate in older age, female sex, and patients with complications of liver cirrhosis like hepatic encephalopathy, acute kidney injury, variceal bleed, and sepsis [4].

Alcohol consumption has increased worldwide and alcohol-related complications range from hepatic steatosis to alcoholic hepatitis to alcoholic cirrhosis and end-stage liver disease [5]. It takes about 10 years to progress, so abstinence is the best way to prevent alcohol-related diseases [6]. According to the World Health Organization (WHO), alcoholism results in 3.3 million deaths every year, which is 6\% of total global annual death [7]. About $10 \%$ to $20 \%$ of patients who are heavy drinkers develop alcohol-related liver diseases [8]. About $36 \%$ of patients with chronic hepatitis have alcohol dependence or abuse problems [9]. Hospital admission in alcoholic liver disease has increased by 18.3\% [10] from 2012 to 2016. Chronic alcoholic liver disease (CALD) is diagnosed with a history of heavy alcohol intake; on physical examination, a patient may have signs Dupuytren's contracture, rhinophyma, spider angioma, palmar erythema, jaundice [8]. In CALD, clinical manifestation starts late and the disease spectrum range from alcoholic hepatitis to alcoholic cirrhosis [11]. 
Various theories are proposed for the pathogenesis of SBP. In patients with liver disease, there is an overgrowth of bacteria in the interstitial lumen [12]. Due to various structural abnormalities like vascular congestion and edema, bacterial translocation occurs in the mesenteric lymph nodes [12]. Patients with liver disease also have decreased immune function leading to bacteria overgrowth and seepage of bacteria into the ascitic fluid [12]. Due to decreased protein levels in cirrhotic patients, low complement level, and poor phagocytic activity, it leads to further growth of bacteria in the ascitic fluid $[13,14]$. SBP contributes about $9.8 \%$ of bacterial infection in alcoholic liver disease [14].

SBP is associated with poor prognosis [12]. Patient survival rates are $67 \%, 50 \%$, and $42 \%$ in one month, six months, and one year, respectively, after an initial diagnosis of SBP [3]. SBP is one of the most common causes of bacterial death that contributes to about $33 \%$ of in-hospital mortality due to infection [3]. SBP occurs most commonly in liver cirrhosis patients [3]. CALD, alcoholic hepatitis, hepatitis B, hepatitis C, hemochromatosis, and autoimmune hepatitis cause liver cirrhosis. One of the studies showed $48 \%$ of patients with liver cirrhosis die because of CALD [8]. So there must be some association between the inhospital mortality in SBP and CALD. The current literature is scarce and contradictory as some studies state that acute kidney injury is the most common cause of in-hospital mortality in SBP patients while some support CALD $[4,15]$.

In our study, we utilized the inpatient data from the United States (US) hospitals: (1) to assess the differences in demographics and comorbidities seen in inpatients for SBP by the presence of comorbid CALD, and (2) to assess the risk of in-hospital mortality due to CALD and other comorbidities in SBP inpatients.

\section{Materials And Methods \\ Data source}

We utilized the Nationwide Inpatient Sample (NIS) data from 2012 to 2014 in our cross-sectional data analysis study. The NIS provides patient health information from about 4,400 non-federal hospitals across 44 states in the US [16]. Diagnostic information in the NIS is detected using the International Classification of Diseases, ninth edition (ICD-9) codes, and Clinical Classification Software (CCS) codes [17].

\section{Inclusion criteria and outcome variables}

We included adult patients (age 18 to 50 years) with a primary discharge diagnosis of SBP using ICD-9 code 567.23. These sample of 6,530 inpatients was further grouped by comorbid discharge diagnosis of CALD ( $N=$ $2,850)$ using the ICD-9 codes 571.2 or 571.3 .

This study included the following demographic variables: age (18-35 and 36-50 years), sex (male or female), and race (white, black, hispanic, and others) [17]. The comorbid diagnosis of diabetes, hypertension, congestive cardiac failure (CCF), AIDS, renal failure, and coagulopathy were identified using ICD-9 or CCS diagnosis codes [17]. We measured the in-hospital mortality between CALD and non-CALD cohorts. In the NIS, in-hospital mortality is reported as all-cause and is the number of inpatient deaths [17].

\section{Statistical analysis}

We used cross-tabulation and descriptive statistics to discern the differences in demographics and comorbidities in SBP inpatients by comorbid CALD. We used a separate model of descriptive statistics to evaluate the in-hospital mortality by comorbidities. Logistic regression analysis was used to measure the odds ratio (OR) association of CALD with in-hospital mortality in SBP inpatients, and the model was controlled for demographic and other chronic comorbidities. A P-value of less than 0.01 was used to determine the statistical significance in all analyses and was conducted using the Statistical Package for the Social Sciences (SPSS), version 26 (IBM Corporation, Armonk, NY).

\section{Ethical approval}

To protect the patient's identity and their health information, the NIS used separate codes to de-identify the database. We do not require approval from the institutional review board [16].

\section{Results}

We analyzed a total sample of 6,530 inpatients hospitalized for SBP, with $43.6 \%$ having comorbid CALD. The majority of the inpatients with CALD were middle-age adults 36 to 50 years (87\%) and were older than the non-CALD cohort (43.3 years vs. 40.6 years, $\mathrm{P}<0.001$ ). A higher proportion of CALD inpatients were male ( $68.8 \%$ vs. $52.6 \%$ ) and white (67\% vs. $51.8 \%$ ) compared to the non-CALD cohort. Comorbidities were seen in a lower proportion of CALD cohort other than a statistically significant difference with comorbid coagulopathy, i.e., $51.2 \%$ in CALD versus $29.8 \%$ in the non-CALD cohort as shown in Table 1. 


\section{Cureus}

\begin{tabular}{|c|c|c|c|c|}
\hline Variable & CALD (-) & CALD (+) & Total & P-value \\
\hline Total (N) & 3680 & 2850 & 6530 & - \\
\hline Mean age, years (SD) & $40.6(8.68)$ & $43.3(5.82)$ & - & $<0.001$ \\
\hline \multicolumn{5}{|l|}{ Age at admission, in \% } \\
\hline 18-35 years & 24.9 & 13.0 & 19.7 & \multirow{3}{*}{$<0.001$} \\
\hline $36-50$ years & 75.1 & 87.0 & 80.3 & \\
\hline \multicolumn{4}{|l|}{ Sex, in \% } & \\
\hline Male & 52.6 & 68.8 & 59.6 & \multirow{2}{*}{$<0.001$} \\
\hline Female & 47.4 & 31.2 & 40.4 & \\
\hline \multicolumn{5}{|l|}{ Race, in \% } \\
\hline White & 51.8 & 67.0 & 58.3 & \multirow{4}{*}{$<0.001$} \\
\hline Black & 20.6 & 4.4 & 13.6 & \\
\hline Hispanic & 19.0 & 20.3 & 19.6 & \\
\hline Other & 8.6 & 8.3 & 8.5 & \\
\hline \multicolumn{5}{|l|}{ Comorbidities, in \% } \\
\hline AIDS & 2.6 & 0.7 & 1.8 & $<0.001$ \\
\hline Diabetes & 14.5 & 13.2 & 13.9 & 0.110 \\
\hline Hypertension & 40.2 & 27.4 & 34.6 & $<0.001$ \\
\hline Congestive cardiac failure & 8.0 & 3.0 & 5.8 & $<0.001$ \\
\hline Coagulopathy & 29.8 & 51.2 & 39.1 & $<0.001$ \\
\hline Renal failure & 29.9 & 11.6 & 21.9 & $<0.001$ \\
\hline In-hospital mortality, in \% & 2.7 & 6.1 & 4.2 & $<0.001$ \\
\hline
\end{tabular}

\section{TABLE 1: Distribution of SBP inpatients by comorbid CALD}

SBP, spontaneous bacterial peritonitis; CALD, chronic alcoholic liver disease

\section{Risk factors for in-hospital mortality in SBP}

Middle-age adults (36-50 years) have 2.8 times higher odds (95\% CI 1.74-4.45) compared to young adults for inpatient death during SBP management. Statistically, no significant impact was seen in in-hospital mortality based on sex distribution $(\mathrm{P}=0.486)$. When compared to the whites, no other races had a statistically significant association with in-hospital mortality.

Among chronic comorbidities, CCF and coagulopathy increased the risk of in-hospital mortality in SBP inpatients by four times (95\% CI 2.46-6.36) and by two times (95\% CI 1.45-2.48), respectively. Hypertension and diabetes had a negative association, whereas AIDS and renal failure had a statistically non-significant association with in-hospital mortality in SBP inpatients. CALD significantly increases the risk of in-hospital mortality by 1.5 times (95\% CI 1.12-1.94) compared to the non-CALD cohort after controlling for demographic and other comorbidities as shown in Table 2. 


\section{Cureus}

\begin{tabular}{|c|c|c|c|c|}
\hline \multirow{3}{*}{ Variable } & \multicolumn{4}{|c|}{ Logistic regression model } \\
\hline & \multirow{2}{*}{ Odds ratio } & \multicolumn{2}{|c|}{$95 \%$ confidence interval } & \multirow{2}{*}{ P-value } \\
\hline & & Lower & Upper & \\
\hline \multicolumn{5}{|l|}{ Age at admission } \\
\hline $18-35$ years & Reference & & & \\
\hline $36-50$ years & 2.78 & 1.74 & 4.45 & $<0.001$ \\
\hline \multicolumn{5}{|l|}{ Sex } \\
\hline Male & 1.10 & 0.84 & 1.46 & 0.486 \\
\hline Female & Reference & & & \\
\hline \multicolumn{5}{|l|}{ Race } \\
\hline White & Reference & & & \\
\hline Black & 0.52 & 0.29 & 0.92 & 0.026 \\
\hline Hispanic & 1.03 & 0.57 & 1.13 & 0.205 \\
\hline Other & 1.03 & 0.66 & 1.60 & 0.895 \\
\hline \multicolumn{5}{|l|}{ Chronic aicoholic liver disease } \\
\hline No & Reference & & & \\
\hline Yes & 1.48 & 1.12 & 1.94 & 0.006 \\
\hline \multicolumn{5}{|l|}{ Comorbidities } \\
\hline No comorbidity & Reference & & & \\
\hline AIDS & 1.77 & 0.69 & 4.55 & 0.236 \\
\hline Diabetes & 0.12 & 0.05 & 0.29 & $<0.001$ \\
\hline Hypertension & 0.48 & 0.33 & 0.69 & $<0.001$ \\
\hline Congestive cardiac failure & 3.96 & 2.46 & 6.36 & $<0.001$ \\
\hline Coagulopathy & 1.89 & 1.45 & 2.48 & $<0.001$ \\
\hline Renal failure & 0.90 & 0.59 & 1.37 & 0.623 \\
\hline
\end{tabular}

TABLE 2: In-hospital mortality risk factors in SBP inpatients

SBP, spontaneous bacterial peritonitis

The highest numbers of death were seen in SBP inpatients with comorbid CALD (63.6\%) followed by coagulopathy (60\%) and diabetes (20\%) as shown in Figure 1. 


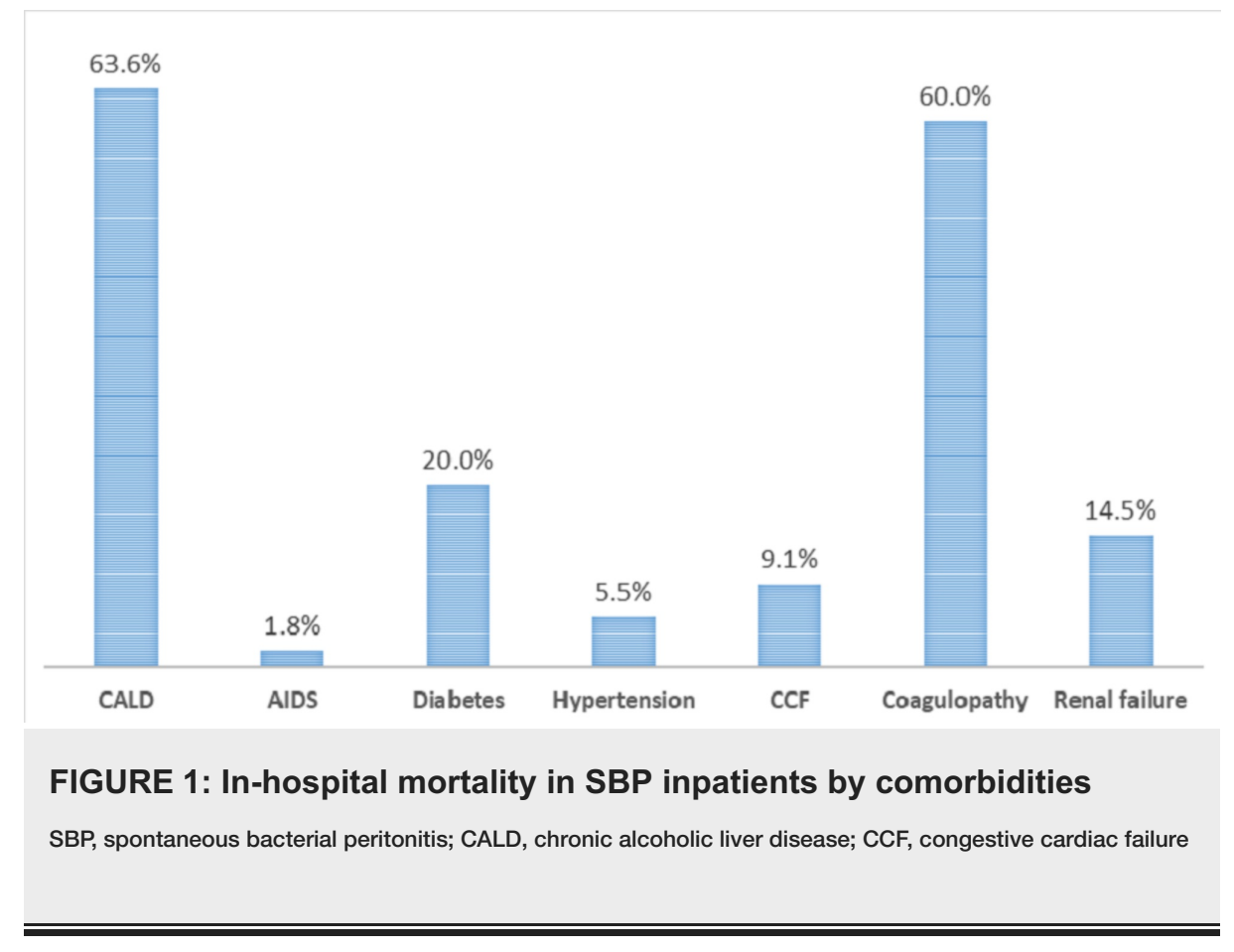

\section{Discussion}

From our analysis conducted from the inpatient data from the US hospitals, the prevalence of CALD in SBP is $43.6 \%$, and the majority of the inpatients were adults age 36 to 50 years, which was correlated to epidemiologic data of alcohol liver disease in Korea [18]. We found that middle-age adults are at a $178 \%$ higher risk of in-hospital mortality during SBP management compared to young adults. Women are at higher risk of developing CALD than men due to environmental and genetic factors [19]. Alcohol consumption and metabolism vary by gender, and so $12-22$ grams/day in women and 24-46 grams/day in men may lead to the development of CALD [20]. About three-fifths of the SBP inpatients with CALD were males, which may be possible as men consume more alcohol than women [21]. We found a non-significant relationship between sex and mortality risk in SBP patients. Our study showed that about $67 \%$ of SBP patients with CALD were whites, which are possibly due to a higher consumption of alcohol in whites compared to other races [22]. There was a statistically non-significant association with mortality risk and race in our study inpatients.

Ethanol metabolism produces reactive oxygen species that in turn activate genes for lipid biosynthesis [23]. The imbalance in the central nervous system (CNS) affects the baroreceptor leading to sympathetic overactivity and stimulation of endothelin [23]. This causes renin-angiotensin activation (RAA), which leads to a decrease in left ventricular contractility [24]. In addition to this, chronic alcoholism leads to cardiomyopathy, myocyte dysfunction, affects calcium homeostasis, malnutrition hence ultimately lead to CCF [24]. Overall, CCF was seen in 5.8\% of SBP inpatients and less in those with CALD (3\%), but CCF was the most prominent risk factor for mortality during SBP hospitalization.

About $60 \%$ of SBP patients with CALD had comorbid coagulopathy, and it was significantly associated with in-hospital mortality and increased the risk by $89 \%$. SBP is common in patients with cirrhosis and ascites, and cirrhotic patients are likely to have an infection that activates cytokines like tumor necrosis factor (TNF)- $\alpha$, interleukin (IL)-6, and IL-1 affecting circulation and coagulation pathway [25]. Therefore, coagulopathy and renal failure further deteriorate CALD. Decompensated CALD is associated with renal failure, due to the reduction of renal blood flow, which leads to vasoconstriction of the main renal and smaller arteries, which further affects cortical ischemia [26]. One of the studies using NIS data showed a 70.3\% in-hospital mortality in SBP patients due to renal failure [4]. But, after controlling for potential confounder in our study, we found a non-significant association between renal failure and mortality risk. We found no significant difference in the prevalence of diabetes between CALD and non-CALD cohorts, and both hypertension and diabetes did not have a positive association with increased in-hospital mortality risk.

Using the multivariable logistic regression model in our study, we found that CALD is significantly associated with increased in-hospital mortality in SBP by $48 \%$. SBP most commonly occurs in a patient with cirrhosis and ascites [27]. Patients with alcoholic cirrhosis are more likely to have ascites, which could contribute to hepatic decompensation in CALD patients, thereby leading to an increase in in-hospital mortality [28].

There are some limitations to our study. Firstly, we used administrative data and so the study inpatients were included based on ICD-9 codes, which may lead to underreporting of comorbid conditions, including 
CALD which may affect the statistical analyses. Second, it includes all-cause in-hospital mortality and so doesn't prove a causal relationship between mortality and comorbidities in SBP. Despite this, we have tried to strengthen our study by using national data, which represents the wide range of hospitals from 44 states across the US and the results are generalized to the inpatient population.

\section{Conclusions}

Medical comorbidities like CCF and coagulopathy increases in-hospital mortality in SBP patients. Chronic alcohol intake leading to CALD was prevalent in SBP patients and was an independent factor that increases the risk of in-hospital mortality by $48 \%$. Alcohol use screening, and alcohol abstinence and supportive therapy need to be implemented at an earlier stage to improve health-related quality of life and reduce inhospital mortality in SBP patients.

\section{Additional Information \\ Disclosures}

Human subjects: Consent was obtained by all participants in this study. Animal subjects: All authors have confirmed that this study did not involve animal subjects or tissue. Conflicts of interest: In compliance with the ICMJE uniform disclosure form, all authors declare the following: Payment/services info: All authors have declared that no financial support was received from any organization for the submitted work. Financial relationships: All authors have declared that they have no financial relationships at present or within the previous three years with any organizations that might have an interest in the submitted work. Other relationships: All authors have declared that there are no other relationships or activities that could appear to have influenced the submitted work.

\section{References}

1. Song DS: Spontaneous bacterial peritonitis. Korean J Gastroenterol. 2018, 72:56-63. 10.4166/kjg.2018.72.2.56

2. Ichou L, Carbonell N, Rautou PE, et al.: Ascitic fluid TREM-1 for the diagnosis of spontaneous bacterial peritonitis. Gut. 2016, 65:536-538. 10.1136/gutjnl-2015-310160

3. Dever JB, Sheikh MY: Review article: spontaneous bacterial peritonitis - bacteriology, diagnosis, treatment, risk factors and prevention. Aliment Pharmacol Ther. 2015, 41:1116-1131. 10.1111/apt.13172

4. Niu B, Kim B, Limketkai BN, Sun J, Li Z, Woreta T, Chen PH: Mortality from spontaneous bacterial peritonitis among hospitalized patients in the USA. Dig Dis Sci. 2018, 63:1327-1333. 10.1007/s10620-0184990-y

5. Hosseini N, Shor J, Szabo G: Alcoholic hepatitis: a review. Alcohol Alcohol. 2019, 54:408-416. 10.1093/alcalc/agz036

6. Hazeldine S, Hydes T, Sheron N: Alcoholic liver disease - the extent of the problem and what you can do about it. Clin Med (Lond). 2015, 15:179-185. 10.7861/clinmedicine.15-2-179

7. Prado V, Caballería J, Vargas V, Bataller R, Altamirano J: Alcoholic hepatitis: how far are we and where are we going?. Ann Hepatol. 2016, 15:463-473. 10.5604/16652681.1202885

8. Singal AK, Bataller R, Ahn J, Kamath PS, Shah VH: ACG clinical guideline: alcoholic liver disease . Am J Gastroenterol. 2018, 113:175-194. 10.1038/ajg.2017.469

9. Rashid W, Patel V, Ravat V, et al.: Problematic cannabis use and risk of complications in patients with chronic hepatitis C. Cureus. 2019, 11:e5373. 10.7759/cureus.5373

10. Hirode G, Saab S, Wong RJ: Trends in the burden of chronic liver disease among hospitalized US adults . JAMA Netw Open. 2020, 3:e201997. 10.1001/jamanetworkopen.2020.1997

11. Farooq MO, Bataller R: Pathogenesis and management of alcoholic liver disease . Dig Dis. 2016, 34:347-355. $10.1159 / 000444545$

12. Marciano S, Díaz JM, Dirchwolf M, Gadano A: Spontaneous bacterial peritonitis in patients with cirrhosis: incidence, outcomes, and treatment strategies. Hepat Med. 2019, 11:13-22. 10.2147/HMER.S164250

13. Toshikuni N, Arisawa T, Tsutsumi M: Nutrition and exercise in the management of liver cirrhosis . World J Gastroenterol. 2014, 20:7286-7297. 10.3748/wjg.v20.i23.7286

14. Park JK, Lee $\mathrm{CH}$, Kim IH, et al.: Clinical characteristics and prognostic impact of bacterial infection in hospitalized patients with alcoholic liver disease. J Korean Med Sci. 2015, 30:598-605. 10.3346/jkms.2015.30.5.598

15. Wiegand J, Kühne M, Pradat P, Mössner J, Trepo C, Tillmann HL: Different patterns of decompensation in patients with alcoholic vs. non-alcoholic liver cirrhosis. Aliment Pharmacol Ther. 2012, 35:1443-1450. 10.1111/j.1365-2036.2012.05108.x

16. Overview of the National (Nationwide) Inpatient Sample . (2019). Accessed: March 15, 2020: https://www.hcup-us.ahrq.gov/nisoverview.jsp.

17. NIS Description of Data Elements . (2019). Accessed: March 15, 2020: https://www.hcupus.ahrq.gov/db/nation/nis/nisdde.jsp.

18. Jang JY, Kim DJ: Epidemiology of alcoholic liver disease in Korea . Clin Mol Hepatol. 2018, 24:93-99. 10.3350/cmh.2017.0079

19. Mueller S, Seitz HK, Rausch V: Non-invasive diagnosis of alcoholic liver disease. World J Gastroenterol. 2014, 20:14626-14641. 10.3748/wjg.v20.i40.14626

20. Jaurigue MM, Cappell MS: Therapy for alcoholic liver disease. World J Gastroenterol. 2014, 20:2143-2158. 10.3748/wjg.v20.i9.2143

21. Basra S, Anand BS: Definition, epidemiology and magnitude of alcoholic hepatitis. World J Hepatol. 2011, 3:108-113. 10.4254/wjh.v3.i5.108 


\section{Cureus}

22. Chartier K, Caetano R: Ethnicity and health disparities in alcohol research. Alcohol Res Health. 2010, 33:152-160.

23. Ansari RA, Husain K, Rizvi SA: Role of transcription factors in steatohepatitis and hypertension after ethanol: the epicenter of metabolism. Biomolecules. 2016, 6:29. 10.3390/biom6030029

24. Laonigro I, Correale M, Di Biase M, Altomare E: Alcohol abuse and heart failure. Eur J Heart Fail. 2009, 11:453-462. 10.1093/eurjhf/hfp037

25. Wong F, Bernardi M, Balk R, et al.: Sepsis in cirrhosis: report on the 7th meeting of the International Ascites Club. Gut. 2005, 54:718-725. 10.1136/gut.2004.038679

26. Domurat ES, Elias AN: The endocrinology and pathophysiology of alcoholic cirrhosis and functional renal failure - a review. J Natl Med Assoc. 1992, 84:153-162.

27. Kraja B, Sina M, Mone I, Pupuleku F, Babameto A, Prifti S, Burazeri G: Predictive value of the model of endstage liver disease in cirrhotic patients with and without spontaneous bacterial peritonitis. Gastroenterol Res Pract. 2012, 2012:539059. 10.1155/2012/539059

28. Hung TH, Tsai CC, Hsieh YH, Tsai CC, Tseng CW, Tseng KC: The effect of the first spontaneous bacterial peritonitis event on the mortality of cirrhotic patients with ascites: a nationwide population-based study in Taiwan. Gut Liver. 2016, 10:803-807. 10.5009/gnl13468 